\title{
Unravelling the Neurobiology of Interoceptive Inference
}

TICS Spotlight on Gehrlach et al (2019) and Livneh et al (2020).

\author{
Micah Allen $1,2,3$ \\ ${ }_{1}$ Aarhus Institute of Advanced Studies, Aarhus University, DK \\ ${ }_{2}$ Center of Functionally Integrative Neuroscience, Aarhus University Hospital, DK \\ ${ }_{3}$ Cambridge Psychiatry, Cambridge University, UK
}

Correspondence: $\underline{\text { micah@cfin.au.dk }}$

Abstract: What is the function of the insular cortex? Two recent studies (Gehrlach et al. and Livneh et al.) offer new insight into how this enigmatic brain region integrates interoception and exteroception in the service of emotion. The findings provide much needed causal evidence for the nascent theory of interoceptive inference.

Two recent studies by Gehrlach et a/ [1] and Livneh et al [2] shed new light on the function of the insular cortex. Early investigations of the insula describe an enigmatic brain region comprised of dense, heterogeneous connections and cell types which seemingly defied simple description [3]. Today, the insula is the subject of intense research interest, implicated in nearly every imaginable cognitive, sensory, and affective domain. Nevertheless, a mechanistic description of the function of the region remains elusive.

Most early theorists described the insula as primary viscero-sensory cortex, encoding internal, physiological changes in heart rate, gastro-intestinal motility, respiration, and arousal. While the relegation of the insula to visceral sensation initially led neuroscientists to assume it was unimportant for higher-order cognition, psychological theory beginning with William James posits that the integration of interoceptive and exteroceptive signals is a core aspect of emotional awareness. On the basis of evidence indicating a multisensory, integrative role for the region, newly popular theories of interoceptive inference argue that the insula compares self-related, top-down predictions with ascending viscerosensory inputs to compute prediction errors that are then used to infer emotional states [4-6]. Until recently, precious little direct evidence existed in support of these proposals. The work of Gehrlach et al and Livneh et al remedies this knowledge gap on at least two crucial points.

Through the application of an innovative range of techniques including optogenetics, cellular-resolution functional imaging, circuit tracing, and clever experimental design, these twin investigations evidence two key postulates of interoceptive inference: (i) that the insula integrates diverse interoceptive and exteroceptive states to facilitate emotional processing; and (ii) that these circuits go beyond the immediate moment to predict future interoceptive states. Although indirect psychophysiological and neuroimaging evidence exists in support of both hypotheses [7,8], thus far little to no direct causal evidence has emerged for the existence of interoceptive predictions or their presumed importance for emotions. 
To investigate the first hypothesis, Gehrlach et a/ [1] applied optogenetics to map the neural circuits by which the posterior insular cortex (pIC) integrates aversive stimuli with interoceptive and emotional states. This enabled the authors to selectively activate or inhibit pIC neurons while rodents underwent noxious exteroceptive or interoceptive stimulation (e.g., electrical shocks or the injection of nausea-inducing lithium chloride) in a variety of behavioral contexts. They showed that optogenetic stimulation of the pIC elicited avoidant, defensive behaviors as well as increased respiratory frequency. When modulating these neurons while mice freely explored a maze, pIC excitation rendered the mice more fearful, whereas inhibition drove the animals to explore more boldly. pIC inhibition further impaired emotional learning, eliminating the association between aversive exteroceptive shocks and negative anxiety-like emotional behavior. Highlighting the dual viscerosensory and integrative role of the $\mathrm{pIC},>$ $99 \%$ of neurons in the region responded to changes in interoception, with $75 \%$ of these exhibiting mixed coding of multiple interoceptive or exteroceptive states. Monosynaptic retrograde tracing further revealed three key insights into pIC connectivity: (i) very high selfconnectivity between insula subregions; (ii) dense, highly correlated reciprocal connectivity with the prefrontal cortex; and (iii) largely unidirectional descending outputs to the amygdala and nucleus accumbens, the activation of which elicited defensive or consummatory behaviors, respectively. These results suggest a hierarchical view of the insula, in which dense multimodal sensory prediction errors converge to guide interoceptive self-inference (Figure 1).

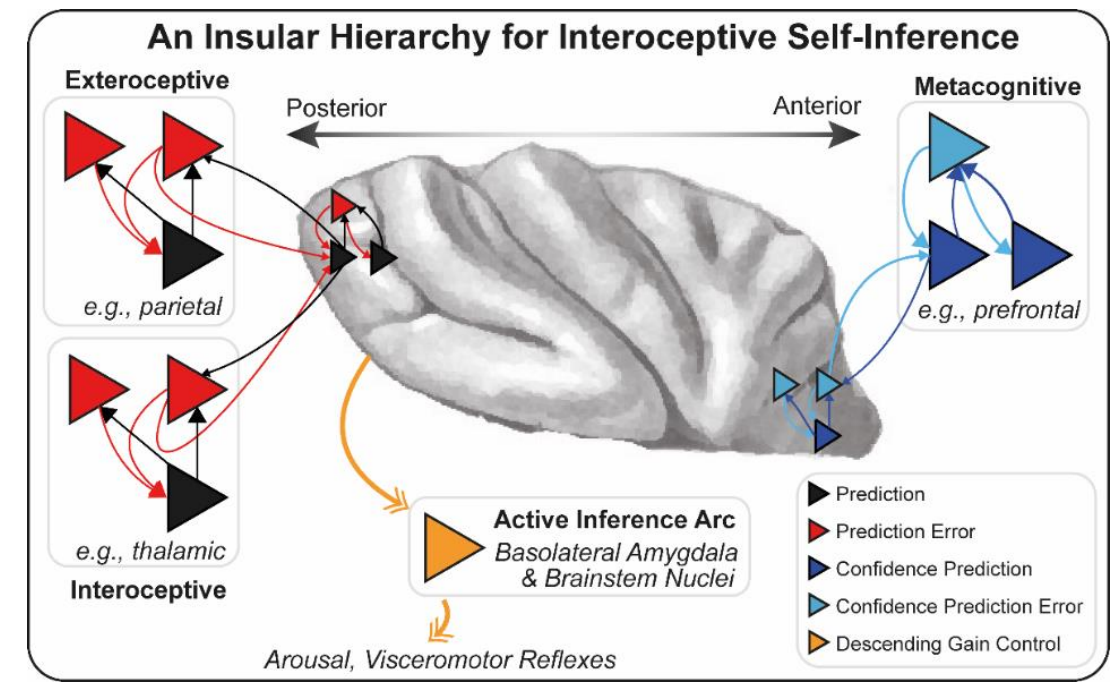

Figure 1 An Insular Hierarchy for Interoceptive Self-inference.

A hypothetical interoceptive hierarchy, synthesizing the findings of Gehrlach et a/[1] and Livneh et al [2] with recent computational models of interoception [10]. Posterior insula receives multimodal innervation encoding ascending exteroceptive and interoceptive prediction errors (PEs). A gradient of interinsula connectivity extracts the confidence, or precision, of these signals to derive a supramodal confidence PE, which is communicated to prefrontal regions to guide metacognition. Unexpected deviations in precision (e.g., as elicited by hyper-arousal) engage active inference through descending neuromodulation of the amygdala and brainstem pathway, to altogether bypass metacognition and trigger embodied visceromotor, allosteric, and emotional reflexes. 
While these results confirm that the insular cortex integrates concurrent emotional, exteroceptive, and interoceptive states, they are silent as to whether the region actually predicts future interoceptive states. To investigate this possibility, Livneh and colleagues [2] applied innovative population-imaging techniques to record insula responses while mice performed a cue-conditioned associative learning task under different interoceptive challenges, such as food or water deprivation. By focusing on not only the cue-evoked neural response, but also the pre-cue ongoing neural activity, Livneh et al. showed that the insula maintains an active representation of visceral states independently of any direct hypothalamic input. Crucially, these neural populations not only signaled current visceral state (e.g., hunger or thirst), but also flipped their pattern of responses when presented with water or food-signaling cues, predicting the upcoming interoceptive state before any actual change in the body. As in the previous study, this linkage of exteroceptive cues and expected interoceptive state was partially mediated by connections between the insula and amygdala. These results demonstrate conclusively that the insula maintains an ongoing representation of interoception and uses this representation to model or simulate future interactions between interoceptive and exteroceptive states.

Collectively, this pair of studies moves the field decisively forward, unravelling the complex multisensory role of the insula. In particular, the results provide decisive causal evidence for the existence of interoceptive predictions and elucidates their role in emotional and interoceptive inference.

Of course, like any well-designed research, the findings also raise intriguing, if difficult to answer questions. Both studies focused on posterior insula, which exhibits a different cytoarchitectural and connectivity profile compared with anterior insula. A recent synthesis of tracing studies in Macaque monkeys suggests that the insula is divided into as many as 16 sub-regions, each with a unique functional profile [9]. A full understanding of the hierarchical organization and function of the region will require investigations that can target, measure, and manipulate these distinct neural populations. Furthermore, while the results provide an exciting first proof of principle that the insula may indeed encode interoceptive predictions, elucidating the exact mechanisms at work will require the integration of neurophysiology and computational modelling. Indeed, while experimentally sensible, both research teams worked hard to exclude a role of autonomic arousal in their results; yet, recent computational models of interoceptive self-inference suggest that descending modulation of arousal is a core mechanism for prioritizing and quenching interoceptive prediction errors [10]. These models also emphasize the importance of interoceptive precision (i.e., confidence) for salience, attention, and metacognition, which is still to be investigated. Future studies will surely build on the results of these two exciting papers further illuminating the neural mechanisms of interoceptive self-inference. 


\section{Acknowledgements}

MA is supported by a Lundbeckfonden Fellowship (R272-2017-4345), and an AIAS-COFUND II fellowship, which is supported by the Marie Skłodowska-Curie actions under the European Union's Horizon 2020 (Grant agreement no 754513), and the Aarhus University Research Foundation. The author thanks Francesca Fardo for useful feedback on the manuscript.

\section{References}

1. Gehrlach, D.A. et al. (2019) Aversive state processing in the posterior insular cortex. Nat. Neurosci. 22, 1424-1437.

2. Livneh, Y. et al. (2020) Estimation of Current and Future Physiological States in Insular Cortex. Neuron, published online January 16, 2020. DOI: 10.1016/j.neuron.2019.12.027

3. Reil, J.C. (1809) Die sylvische Grube. Arch Physiol 9, 195-208.

4. Seth, A.K. (2013) Interoceptive inference, emotion, and the embodied self. Trends Cog Sci 17, 565-573.

5. Allen, M. and Friston, K.J. (2018) From cognitivism to autopoiesis: towards a computational framework for the embodied mind. Synthese 195, 2459-2482

6. Barrett, L.F. and Simmons, W.K. (2015) Interoceptive predictions in the brain. Nat Rev Neurosci 16, 419-429.

7. Allen, M. et al. (2016) Anterior insula coordinates hierarchical processing of tactile mismatch responses. Neurolmage 127, 34-43.

8. Allen, M. et al. (2016) Unexpected arousal modulates the influence of sensory noise on confidence. elife 5, e18103.

9. Evrard, H.C. (2019) The Organization of the Primate Insular Cortex. Front. Neuroanat. 13, 2019, 43.

10. Allen, M. et al. (2019) In the Body's Eye: The Computational Anatomy of Interoceptive Inference, bioRxiv 2019, Published online April 10, 2019. https://doi.org/10.1101/603928 